\title{
Neuro-Oncology Clinicians' Attitudes and Perspectives on Medical Assistance in Dying
}

Seth Andrew Climans ( $\nabla$ seth.climans@gmail.com )

University of Toronto https://orcid.org/0000-0002-4961-237X

Warren P Mason

University of Toronto

Kim Edelstein

University of Toronto

Caroline Variath

University of Toronto

Jennifer A H Bell

University of Toronto

\section{Research Article}

Keywords: Medical assistance in dying, End-of-life care, Brain cancer, Decision-making capacity

Posted Date: April 16th, 2021

DOI: https://doi.org/10.21203/rs.3.rs-422892/v1

License: (c) (i) This work is licensed under a Creative Commons Attribution 4.0 International License.

Read Full License 


\section{Abstract}

Introduction

Medical assistance in dying (MAiD), also known as physician-assisted death, is currently legal in several locations across the globe. Even more jurisdictions are considering legalizing MAiD. Brain cancer or its treatments can lead to cognitive impairment, which can impact decision-making capacity. In most jurisdictions patients are assessed for mental capacity to inform MAiD eligibility. We sought to explore worldwide neuro-oncology clinicians' attitudes and perspectives on MAiD, including interpretation of decision-making capacity for patient MAiD eligibility.

Methods

An online survey was distributed to members of national and international neuro-oncology societies. We asked questions about decision-making capacity and MAiD, in part using hypothetical patient scenarios. Multiple choice and free-text responses were captured.

Results

There were 125 survey respondents. Impaired cognition was identified as the most important factor that would signal a decline in patient capacity. At least $26 \%$ of survey respondents had moral objections to MAiD. Fewer clinicians were willing to support a MAiD decision for patients with lower-grade tumors and better performance status.

\section{Conclusions}

hile there are differing opinions on the moral permissibility of MAiD in general and for neuro-oncology patients, most clinicians agree that capacity must be assessed carefully before a decision is made. Further, some patients with brain tumors are not generally thought to be MAiD-eligible due to the nature of their specific diseases. These results can inform assessments of patient capacity in neuro-oncological practice in jurisdictions where MAiD is legal.

\section{Background}

Medical assistance in dying (MAiD), also known as physician-assisted death, is currently legal in several countries across the globe, including Canada, Belgium, the Netherlands, Luxembourg, Colombia, and Switzerland [1, 2]. It is also legal in several American states [1] and in one Australian state [3]. All of these jurisdictions have established patient eligibility criteria for MAiD to ensure appropriate access and safeguards [1]. Many locations also require that two independent physicians make the determination of MAiD eligibility [1]. MAiD can include either assisted suicide or voluntary euthanasia. Criteria often include being a competent adult suffering from a medical condition likely to cause their death though in some places, advanced directives are possible and in some places reasonably-foreseeable natural death is not required [1]. 
Previous research has demonstrated that many individuals who request MAiD are patients with advanced and palliative-stage cancer [4-6]. Of those who are very near death, continuation of their decision-making capacity is a concern since in most jurisdictions patients must be competent to provide informed consent at the time of MAiD intervention. Competency is a legal construct that involves patients having the capacity to make an informed decision. This includes having the ability to understand and appreciate the benefits and risks of a proposed treatment or intervention, the alternatives to those treatments or interventions, including receiving no disease-directed treatment or intervention [7].

Brain cancer poses a unique threat to patients' decision-making capacity. Brain cancer includes primary brain tumors and metastases to the brain. Depending on the location of the cancer, individual lesions or combinations of lesions may infringe upon aspects of language, memory, and executive functions considered central to patient identity and capacity to make healthcare decisions. As a direct result of their disease, some patients may lose capacity to choose MAiD, thus becoming ineligible to access this intervention. Since the desire to maintain control and autonomy influence MAiD requests [8, 9], patient motivation for MAiD may be high in this population, while at the same time these patients may face barriers to accessing MAiD due to the nature of their specific disease.

Clinical interpretation of decision-making capacity is important for determinations of MAiD eligibility. Determinations of MAiD eligibility intersect with ethical issues such as equitable access, power differences, respect for autonomy, fairness, and protection of the vulnerable [10]. Healthcare providers are often the intermediary between patient requests for MAiD and access to this intervention. Therefore, it is important to understand how providers interpret the impact of brain cancer on patient capacity to make end-of-life decisions including MAiD. Although some articles have examined the impact of brain cancer on decision-making capacity $[11,12]$, none have so far specifically looked at capacity for a MAiD decision in these patients. Capacity is decision-specific and different decisions may require greater or lesser cognitive function. Making a decision around MAiD might constitute a different type of decision since it is an active choice to end one's life motivated by illness. The purpose of this study was to explore neurooncology clinicians' attitudes and perspectives on MAiD, including interpretation of decision-making capacity for patient MAiD eligibility.

\section{Methods}

Following local research ethics board approval, an international online survey was conducted with members of Society for Neuro Oncology (SNO), European Association of Neuro-Oncology (EANO), the Oncology Special Interest Group of the International Neuropsychological Society (INS SIG), and a Canadian neuro-oncology mailing list from September to December 2020. Consent to participate was implied by completion of the survey. The survey had several sub-sections and was developed by the research team to understand (1) clinician characteristics and demographics, (2) their stance on MAiD, (3) cognitive factors that they believe underlie capacity, and (4) interpretations of hypothetical scenarios. The full questionnaire can be found in the Supplementary Material. Nine scenarios of brain cancer patients requesting MAiD were presented. The scenarios were informed by our clinical practice and created to 
explore the challenges of assessing the nuances presented by patients' varying degrees of cognitive impairment. The scenarios also explored opinions about patient MAiD eligibility under varying clinical situations. The first four scenarios differed with regards to clinical features that might impact their capacity. Respondents were asked whether they believe the patient would have the capacity to make this decision. The last five scenarios described patients with differing age, tumor type, performance status, and time since diagnosis. Respondents were asked whether, if medical assistance in dying (MAiD) were legally available in their jurisdiction, they would support the patient's decision to access MAiD. A free text option was included to allow for elaboration. Survey data were collected and managed using REDCap electronic data capture tools hosted at University Health Network [13, 14]. At the end of the survey, respondents were asked to leave their contact information if they were interested in participating in a follow-up interview. Data from these interviews will be published separately.

\section{Data Collection and Analysis}

The survey was distributed to a list of Canadian neuro-oncology clinicians, to members of SNO, EANO, and the INS SIG (Fig. 1). Data were analyzed using R [15]. Exploratory logistic and ordinal regression analyses, respectively, were conducted to determine clinician predictors of (1) moral opposition to MAiD, and (2) number of clinical scenarios felt to be either capable or MAiD-eligible, depending on the scenario. For the MAiD scenarios, we conducted pairwise comparisons of proportions using Fisher's exact test. Because multiple comparisons were conducted, P-values $\leq 0.01$ were considered to be statistically significant. Nonetheless, there are differences in opinion regarding the appropriate a level correction [16, 17] and results should be interpreted with caution. Thematic analysis was applied to the free-text fields, where the researchers (SAC, JAHB) read through the replies to familiarize themselves with the data and gain an overall perspective of responses. Paying close attention to the data, similar descriptions and phrases were then identified and categorized according to codes. Codes were collapsed into broader themes and relationships between themes were explored [18].

\section{Results}

\section{Responses}

The survey was sent to more than 10,000 people and 133 responded, 125 of whom were neuro-oncology clinicians (Fig. 1).

\section{Clinician Demographics and Characteristics}

Most respondents were neuro-oncologists (57\%), including medical oncologists who treat brain tumors. Other represented professionals included neurosurgeons, radiation oncologists, advanced practice providers, psychologists and neuropsychologists, medical trainees, nurses, palliative care physicians, other physicians, research coordinators, and social workers (Table 1). Christianity was the most common religious group (45\%) but the no religion or no response group was $43 \%$. Within the Christian group, Catholic faith was the most common (52\%) followed by unspecified, Protestant, and Eastern Orthodox. 
Survey responses came in from around the world aincluding six continents (Fig. 2). Most responses came from the United States, followed by Canada, the Netherlands, and Italy.

\section{Attitudes towards MAiD}

A minority (39\%) of respondents worked in a jurisdiction where MAiD is legal. Only $16 \%$ of respondents had participated in the MAiD process, either as an assessor or by prescribing medications. One quarter (26\%) of respondents held moral objections to MAiD while $57 \%$ did not and the remaining $17 \%$ were unsure.

Using a logistic regression analysis, factors found to make a respondent's moral objection to MAiD significantly more likely included (1) shorter time in practice, (2) working in a country other than the United States, Canada, or the Netherlands (3) Christian faith, as compared to no religion (Supplementary Table 3). The longer neuro-oncology clinicians have been in practice, the less likely they are to have moral objections to MAiD. Notably, factors that did not predict moral objection to MAiD included profession, gender, Catholicism, age of patients, clinical volume, and whether MAiD is allowed regionally.

\section{Cognitive Factors Underlying Capacity}

Survey respondents were asked to rank the three most important Diagnostic and Statistical Manual of Mental Disorders cognitive domains necessary for patient capacity for consenting to MAiD [19]. Executive function was ranked most important by $41 \%$ of respondents, followed by complex attention (21\%), language (14\%), and learning and memory (14\%).

Analysis of the free-text responses revealed more detail. Impaired cognition was identified by a majority of respondents as one of the most important factors that would signal a decline in patient capacity, e.g. "when their cognitive functions are significantly impaired". Respondents understood impaired cognition as including patients' lack of understanding of treatments, the consequences of treatments, and lack of insight or awareness of the situation or options for care. Impaired language and impaired cognition in general was also highlighted by many respondents as influencing capacity. Impaired language was viewed as the inability to understand information and inability to recall and communicate information about intervention choices. One respondent wrote that incapacity should be suspected when "they can't communicate their reasons for making one choice vs another." Additionally, impaired short-term memory, confusion, and disorientation were identified as factors that may signal a decline in patient capacity. Taken together, these factors became very important when identified as severe or persistent, such as when there is "severe decline in memory or cognitive status." Respondents remarked on how these limitations were complex, difficult to assess, and that capacity was individualized. Some reflected on patients who are able to contribute meaningfully to their care and who can articulate their understanding and wishes. Incapacity then became a sense of when conversations with the patient "no longer have depth" or the person cannot communicate their reasons for choice. 
Incapacity was seen as more likely to occur in patients living with advanced disease or who were actively dying. Respondents became concerned about a patient's potential incapacity when there was disease progression or poor scores on objective capacity tests. Comorbid conditions, such as depression, and medication use (e.g, steroids) were also flagged as potential negative influences on capacity. Some respondents identified family as a resource that could provide collateral information to help clinicians assess patient capacity.

\section{Interpretations of Hypothetical Scenarios with Questionable Capacity}

Four capacity scenarios were presented to survey respondents. Hypothetical female glioblastoma patients with deficits in language, memory, wakefulness, or personality were described. Respondents were asked whether they believe the patient has the capacity to decide whether to opt for MAiD. Between $18 \%$ and $58 \%$ of respondents, depending on the scenario, thought that the patient had the capacity for this choice (Fig. 3A). Many respondents used the accompanying textbox to elaborate on their responses, and specifically to highlight the complexity of the scenarios and the limitations of being asked to provide yes/no answers. Some wrote that there was "not enough information to judge." Most respondents believed that in-depth-capacity assessments were necessary for these scenarios. An assessment of the extent of neurological deficit was thought to be required to ascertain how the aphasia, amnesia, somnolence, or personality changes might impact the ability to understand, appreciate, and make a decision that aligned with previously-expressed patient values. Some questioned whether the requests for MAiD were consistent over time and whether they were aligned with patients' prior expressed wishes. Respondents believed that patients could retain decision-making capacity despite short-term memory loss, somnolence, and personality changes, if patients could demonstrate consistent and stable choices. Some commented that we must determine whether patients can "make rational decisions that reflect their values." Input from family, where available, was thought to be helpful in assessing these factors for patient capacity.

Respondents gave explanations for why they did or did not believe each hypothetical patient had the capacity to decide whether to opt for MAiD. Representative quotes in favour of or against capacity for each scenario are shown in Table 2. Using an ordinal regression analysis, two factors were found to predict the belief that patients have decision-making capacity for MAiD. Advanced practice practitioners (versus neuro-oncologists) and Canadians (versus Americans) were more likely to believe that the four hypothetical patients had decision-making capacity (Supplementary Table 1).

\section{Interpretations of Hypothetical Scenarios with Varying Clinical Factors}

Five MAiD scenarios were presented to survey respondents. The scenarios varied the hypothetical male glioma patient's age, tumor grade, time since diagnosis, and performance status. Respondents were asked whether they would support the patient's decision to access MAiD, assuming it was legally available. Between $26 \%$ and $78 \%$ of respondents, depending on the scenario, supported the patient's MAiD decision (Fig. 3B). Many respondents used the accompanying textbox to explain that the scenarios lacked sufficient information to make this judgement. They emphasized the importance of proper 
capacity assessment, neuropsychological testing, and ruling out depression. They wanted to make sure that each patient properly understands their treatment options and prognosis.

When clinicians did not support a patient's MAiD decision, they wrote several explanations. Some felt that palliative care is a better option. Others cited moral objections. Some scenarios involved patients one week after diagnosis. In these situations, many felt that this was too soon after diagnosis to make a rational decision for MAiD. Others felt that it was too soon to establish rapport with the patient to know if their choice was consistent over time. In the oligodendroglioma case, many felt that death was not reasonably foreseeable and that too many treatment options were still available. Young age was sometimes brought up as a positive prognostic factor, and that in such patients, symptoms might improve with time, which cast doubt on clinicians' belief that MAiD would be an acceptable option for these patients. When clinicians did support a patient's MAiD decision, they generally emphasized patient autonomy. They noted that all scenarios outlined patients with incurable conditions. Some with stated moral objections to MAiD were willing to refer to a non-objecting provider.

Using an ordinal regression analysis, we found three factors that predict support for MAiD in the scenarios. Non-American, non-Canadian, and non-Dutch (versus American) and Canadian (versus American) clinicians were less likely to support a patient's decision to access MAiD (Supplementary Table 2). Canadian (versus American) clinicians and advanced practice providers (versus neuro-oncologists) were more likely to support a patient's decision to access MAiD. Pairwise comparison of scenarios 5 versus 6,5 versus 8 , and 8 versus 9 demonstrated the importance of performance status, time since diagnosis, and age, respectively. The P-values for these comparisons were $0.007,<0.001$, and 0.049 . Clearly, time since diagnosis, performance status, and patient age are all important factors when choosing whether to support a patient's request for MAiD. Tumor grade is presumably important, but limitations in the scenarios did not allow pairwise comparison over this variable.

\section{Discussion}

Our mixed-methods analysis has revealed the opinions of some neuro-oncology clinicians on decisionmaking capacity around MAiD, their moral stances toward MAiD, and degree of support for different patients' MAiD requests. While there are differing attitudes about the moral acceptability of MAiD in general, and for neuro-oncology patients in particular, most clinicians agree that patient capacity must be assessed carefully. Some neuro-oncology patients are not thought to be MAiD-eligible due to lack of capacity or lack of reasonably foreseeable natural death.

Our study has some important strengths. We surveyed clinicians from around the world, addressed a timely yet understudied topic, and examined this issue with relevance for a particularly vulnerable patient population. The survey included clinicians of diverse backgrounds and opinions. Detailed interviews with our respondents are currently in progress. These should better illuminate the attitudes and perspectives of the neuro-oncology community on the topic of MAiD. 
Decisions around end-of-life care are very significant, since they involve understanding complex medical information and may result in death, an irreversible state. When brain cancer patients lose their decisionmaking capacity, it is usually in their last month of life [20]. In a retrospective cohort of 101 Dutch glioma patients, $80 \%$ retained capacity in the last months of life, $47 \%$ retained capacity in the last weeks of life, and $14 \%$ retained capacity in the last days of life [20]. Clinicians involved in the care of neuro-oncology patients must be able to recognize when decision-making capacity has been lost. Consequently, it is critical to engage each patient in discussions around end-of-life care before it is too late. Our results show that neuro-oncology clinicians are more likely to support a patient's end-of-life request if it is expressed consistently. This is even more reason to have end-of-life discussions early, before capacity is lost. There are several end-of-life decisions that might need to occur with input from the patient: specifying the optimum location for end-of-life care, whether hospice, hospital, or home; balancing the need for symptom control and desired level of alertness, since pain and seizure medication can be sedating; and lastly, whether MAiD or palliative sedation is clinically appropriate and aligned with patient values.

The main limitation of this study is the risk for sampling bias, since we sent survey invitations to more than 10,000 subjects, but only had survey responses from 125 . This is lower than other recent SNO surveys that had between 426 and 480 responses [21,22]. The low participation rate in our study may in part be due to the inclusion of non-clinicians on the mailing lists of neuro-oncological societies, the controversial topic of the survey, and the fact that we did not send out reminder emails. Our questionnaire scenarios forced a yes/no response with limited presented information, which created a forced dichotomy out of otherwise nuanced decisions. This may have reduced the reliability of our scenario responses, however, the option for free-text and our follow-up interviews to be published separately aim to capture and describe these nuances. Some clinicians who answered the survey treat only pediatric patients, so they might not have been able to rely on clinical experience to respond to the presented case scenarios. For the regression analyses, many of the subgroups were small, so these predictors can only be hypothesis-generating and not definitive.

Despite the limitations, our study results may help inform best-practice guidelines for clinical assessments of MAiD decision-making capacity in neuro-oncology patients. Our findings will inform teaching tools and webinars on this topic and may help create instruments for assessing decisionmaking capacity. Disease experts can play an important role in both capacity assessment and predictions about prognosis. Each MAiD assessment ought to be individualized, but an understanding of the neuro-oncology community's attitudes and perspectives on the matter can guide our care.

\section{Declarations}

Funding: This research is supported by the Division of Palliative Medicine, Department of Medicine, University of Toronto; the Dalla Lana School of Public Health, University of Toronto; the Global Institute of Psychosocial, Palliative and End-of-Life Care (GIPPEC); the Princess Margaret Cancer Foundation, and the Ontario Ministry of Health and Long Term Care. The views expressed are not necessarily those of the OMOHLTC. 
Conflicts of interest/Competing interests: The authors have no relevant financial or non-financial interests to disclose.

Availability of data and material: Available upon reasonable request.

Code availability: Available upon reasonable request.

Authors' contributions: All authors contributed to the study conception and design. Material preparation, data collection and analysis were performed by Seth Climans, Kim Edelstein, Caroline Variath, Warren Mason, and Jennifer Bell. The first draft of the manuscript was written by Seth Climans and all authors commented on previous versions of the manuscript. All authors read and approved the final manuscript.

Ethics approval: The questionnaire and methodology for this study was approved by the human research ethics committee of the University Health Network (Ethics approval number: 20-5115).

\section{Acknowledgements}

This research is supported by the Division of Palliative Medicine, Department of Medicine, University of Toronto; the Dalla Lana School of Public Health, University of Toronto; the Global Institute of Psychosocial, Palliative and End-of-Life Care (GIPPEC); the Princess Margaret Cancer Foundation, and the Ontario Ministry of Health and Long Term Care. The views expressed are not necessarily those of the OMOHLTC.

\section{References}

1. Emanuel EJ, Onwuteaka-Philipsen BD, Urwin JW, Cohen J (2016) Attitudes and practices of euthanasia and physician-assisted suicide in the United States, Canada, and Europe. JAMA 316:79. https://doi.org/10.1001/jama.2016.8499

2. Li M, Watt S, Escaf M, et al (2017) Medical assistance in dying-implementing a hospital-based program in Canada. N Engl J Med 376:2082-8

3. Duckett S (2019) The long and winding road to assisted dying in Australia. Aust J Soc Issues 54:386-400. https://doi.org/10.1002/ajs4.77

4. Ruijs CD, Kerkhof AJ, van der Wal G, Onwuteaka-Philipsen BD (2013) Symptoms, unbearability and the nature of suffering in terminal cancer patients dying at home: a prospective primary care study. BMC Fam Pract 14:201. https://doi.org/10.1186/1471-2296-14-201

5. Steck N, Egger M, Maessen M, et al (2013) Euthanasia and assisted suicide in selected European countries and US states: systematic literature review. Med Care 51:938-944. https://doi.org/10.1097/MLR.0b013e3182a0f427

6. Downar J, Fowler RA, Halko R, et al (2020) Early experience with medical assistance in dying in Ontario, Canada: a cohort study. CMAJ 192:E173-E181. https://doi.org/10.1503/cmaj.200016 
7. Leo RJ (1999) Competency and the capacity to make treatment decisions: A primer for primary care physicians. Prim Care Companion J Clin Psychiatry 1:131-141

8. Eliott JA, Olver IN (2008) Dying cancer patients talk about euthanasia. Soc Sci Med 1982 67:647656. https://doi.org/10.1016/j.socscimed.2008.04.004

9. Hendry M, Pasterfield D, Lewis R, et al (2013) Why do we want the right to die? A systematic review of the international literature on the views of patients, carers and the public on assisted dying. Palliat Med 27:13-26. https://doi.org/10.1177/0269216312463623

10. Meisel A (2005) Ethics and law: Physician-assisted dying. J Palliat Med 8:609-621. https://doi.org/10.1089/jpm.2005.8.609

11. Hewins W, Zienius K, Rogers JL, et al (2019) The Effects of Brain Tumours upon Medical DecisionMaking Capacity. Curr Oncol Rep 21:55. https://doi.org/10.1007/s11912-019-0793-3

12. Pace A, Koekkoek JAF, van den Bent MJ, et al (2020) Determining medical decision-making capacity in brain tumor patients: Why and how? Neuro-Oncol Pract 7:599-612. https://doi.org/10.1093/nop/npaa040

13. Harris PA, Taylor R, Thielke R, et al (2009) Research electronic data capture (REDCap)-A metadatadriven methodology and workflow process for providing translational research informatics support. J Biomed Inform 42:377-381. https://doi.org/10.1016/j.jbi.2008.08.010

14. Harris PA, Taylor R, Minor BL, et al (2019) The REDCap consortium: Building an international community of software platform partners. J Biomed Inform 95:103208. https://doi.org/10.1016/j.jbi.2019.103208

15. R Core Team (2018) R: A Language and Environment for Statistical Computing. R Foundation for Statistical Computing, Vienna, Austria

16. Perneger TV (1998) What's wrong with Bonferroni adjustments. BMJ 316:1236-1238. https://doi.org/10.1136/bmj.316.7139.1236

17. Simes RJ (1986) An improved Bonferroni procedure for multiple tests of significance. Biometrika 73:751-754. https://doi.org/10.1093/biomet/73.3.751

18. Braun V, Clarke V (2006) Using thematic analysis in psychology. Qual Res Psychol 3:77-101. https://doi.org/10.1191/1478088706qp063oa

19. American Psychiatric Association (2013) Diagnostic and Statistical Manual of Mental Disorders, Fifth Edition. American Psychiatric Association

20. Sizoo EM, Pasman HR, Buttolo J, et al (2012) Decision-making in the end-of-life phase of high-grade glioma patients. Eur J Cancer 48:226-32. https://doi.org/10.1016/j.ejca.2011.11.010

21. Taylor JW, Armstrong T, Kim AH, et al (2019) The lomustine crisis: Awareness and impact of the 1500\% price hike. Neuro-Oncol 21:1-3. https://doi.org/10.1093/neuonc/noy189

22. Rogers JL, Acquaye A, Vera E, et al (2020) Provider-reported challenges and barriers to referring patients to neuro-oncology clinical trials: a report from the Society for Neuro-Oncology member survey. Neuro-Oncol Pract 7:38-51. https://doi.org/10.1093/nop/npz038 


\section{Tables}

Table 1: Respondent characteristics 


\begin{tabular}{|c|c|c|}
\hline Characteristic & Classification & Count (\%) \\
\hline \multirow[t]{7}{*}{ Profession } & Neuro-oncologist & $71(57)$ \\
\hline & Neurosurgeon & $17(14)$ \\
\hline & Radiation oncologist & $11(9)$ \\
\hline & Advanced practice provider & $10(8)$ \\
\hline & Psychologist & $4(3)$ \\
\hline & Medical trainee & $4(3)$ \\
\hline & Other & $8(6)$ \\
\hline \multirow[t]{2}{*}{ Gender } & Female & $62(50)$ \\
\hline & Male & $63(50)$ \\
\hline \multirow[t]{3}{*}{ Population they treat } & Adults & $95(76)$ \\
\hline & Children & $11(9)$ \\
\hline & Both & $19(15)$ \\
\hline \multirow[t]{5}{*}{ Years in practice } & $0-4$ years & $22(18)$ \\
\hline & $5-9$ years & $22(18)$ \\
\hline & $10-14$ years & $27(22)$ \\
\hline & $15-19$ years & $18(14)$ \\
\hline & $20+$ years & $36(29)$ \\
\hline \multirow[t]{6}{*}{ Brain tumor patient volume } & 0-1 per week & $7(6)$ \\
\hline & $1-2$ per week & $12(10)$ \\
\hline & $3-10$ per week & $39(31)$ \\
\hline & 11-20 per week & $39(31)$ \\
\hline & $21-50$ per week & $25(20)$ \\
\hline & $51+$ per week & $3(2)$ \\
\hline \multirow[t]{5}{*}{ Religion (if any) } & Christian & $56(45)$ \\
\hline & No religion & $41(33)$ \\
\hline & Jewish & $8(6)$ \\
\hline & Muslim & $4(3)$ \\
\hline & Other & $3(2)$ \\
\hline
\end{tabular}

Page 12/16 
Table 2: Explanations of responses to the capacity scenarios

\begin{tabular}{|c|c|c|}
\hline Scenario & $\begin{array}{l}\text { Thought } \\
\text { to be } \\
\text { capable }\end{array}$ & Representative quote \\
\hline \multirow[t]{2}{*}{ Aphasia } & Yes & $\begin{array}{l}\text { "With careful explanation, I believe the patient may be found to be able } \\
\text { to consent" }\end{array}$ \\
\hline & No & "If patient cannot understand risk, then really can't consent" \\
\hline \multirow[t]{2}{*}{ Amnesia } & Yes & $\begin{array}{l}\text { "They can usually sustain enough memory to be able to grasp the } \\
\text { concepts at this stage" }\end{array}$ \\
\hline & No & "She has severe dementia and/or delirium based on the vignette" \\
\hline \multirow[t]{2}{*}{ Somnolence } & Yes & $\begin{array}{l}\text { "Maybe - if she is able to have discussion and give consent for MAID - } \\
\text { may have to be in short conversations to work around her fatigue" }\end{array}$ \\
\hline & No & $\begin{array}{l}\text { "She does not seem alert enough to be able to participate in discussions } \\
\text { or the process itself" }\end{array}$ \\
\hline \multirow{2}{*}{$\begin{array}{l}\text { Personality } \\
\text { change }\end{array}$} & Yes & "Behavior disturbances do not mean lack of mental capacity" \\
\hline & No & $\begin{array}{l}\text { "If she is genuinely irrational she cannot make a proper assessment of } \\
\text { her situation" }\end{array}$ \\
\hline
\end{tabular}

\section{Figures}

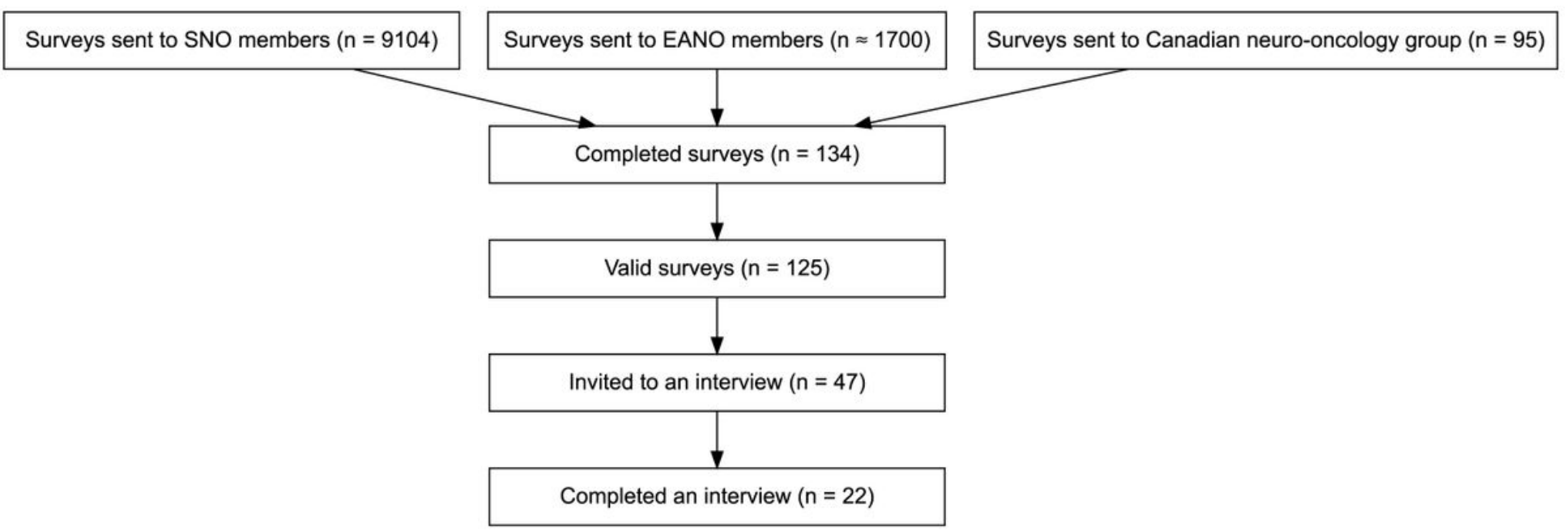

\section{Figure 1}

Flow chart of survey invitations, responses, and interviews. Invitations may have been sent to the same person more than once where that person is a member of multiple neuro-oncological societies (Society 
for Neuro-Oncology, European Association of Neuro-Oncology, Canadian neuro-oncology mailing list, and International Neuropsychological Society oncology Special Interest Group). Eight respondents started the survey but indicated they are not involved in the clinical care of patients, so they did not complete the survey. One survey response was duplicate

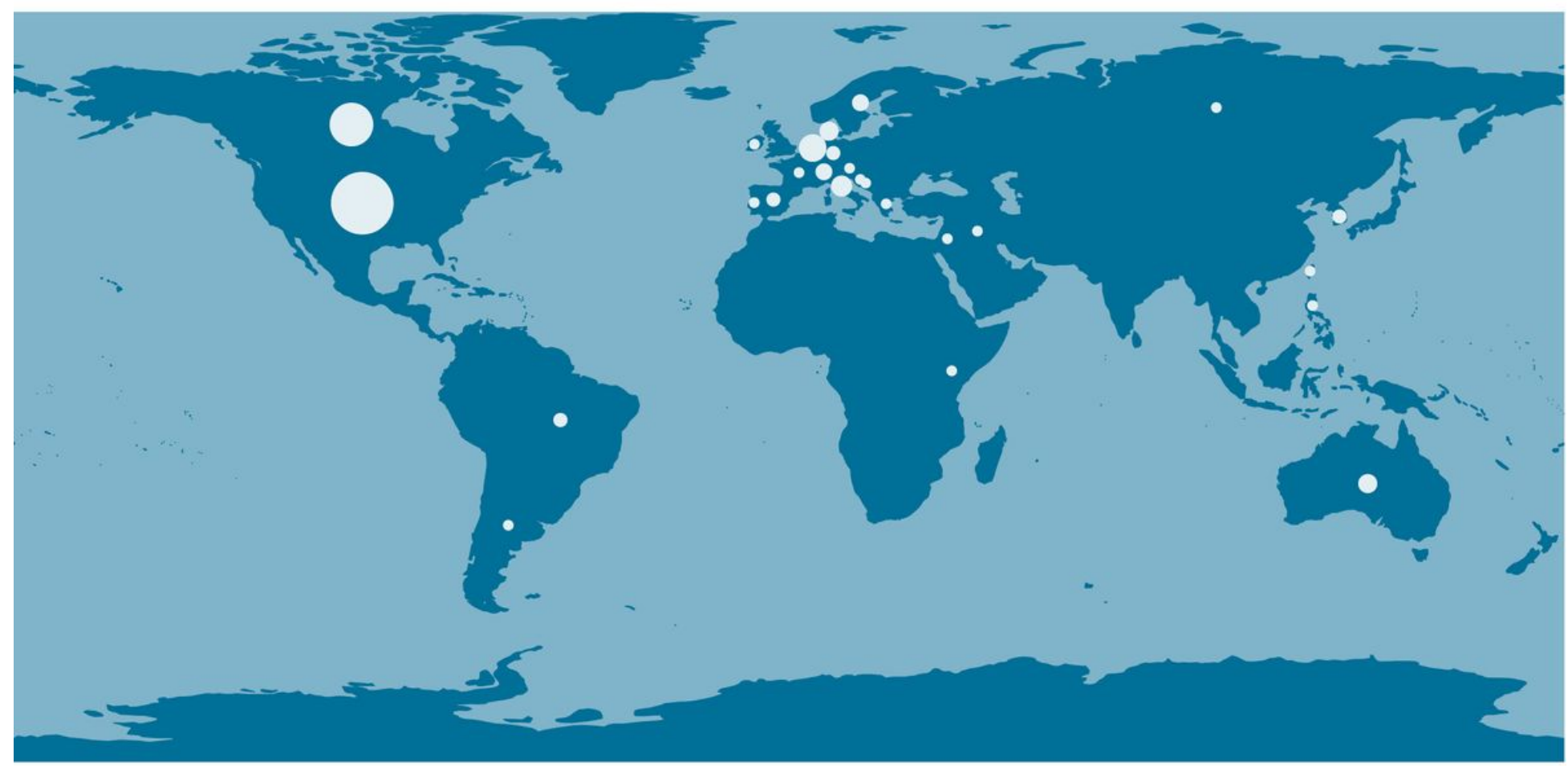

\section{Count}

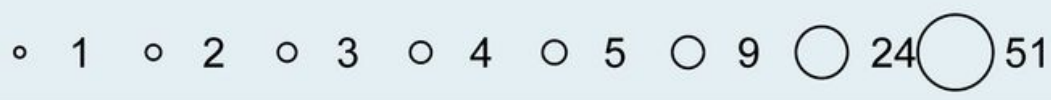

\section{Figure 2}

Map of survey responses by country. Note: The designations employed and the presentation of the material on this map do not imply the expression of any opinion whatsoever on the part of Research Square concerning the legal status of any country, territory, city or area or of its authorities, or concerning the delimitation of its frontiers or boundaries. This map has been provided by the authors. 
Do you believe she will have the mental capacity to make this decision?

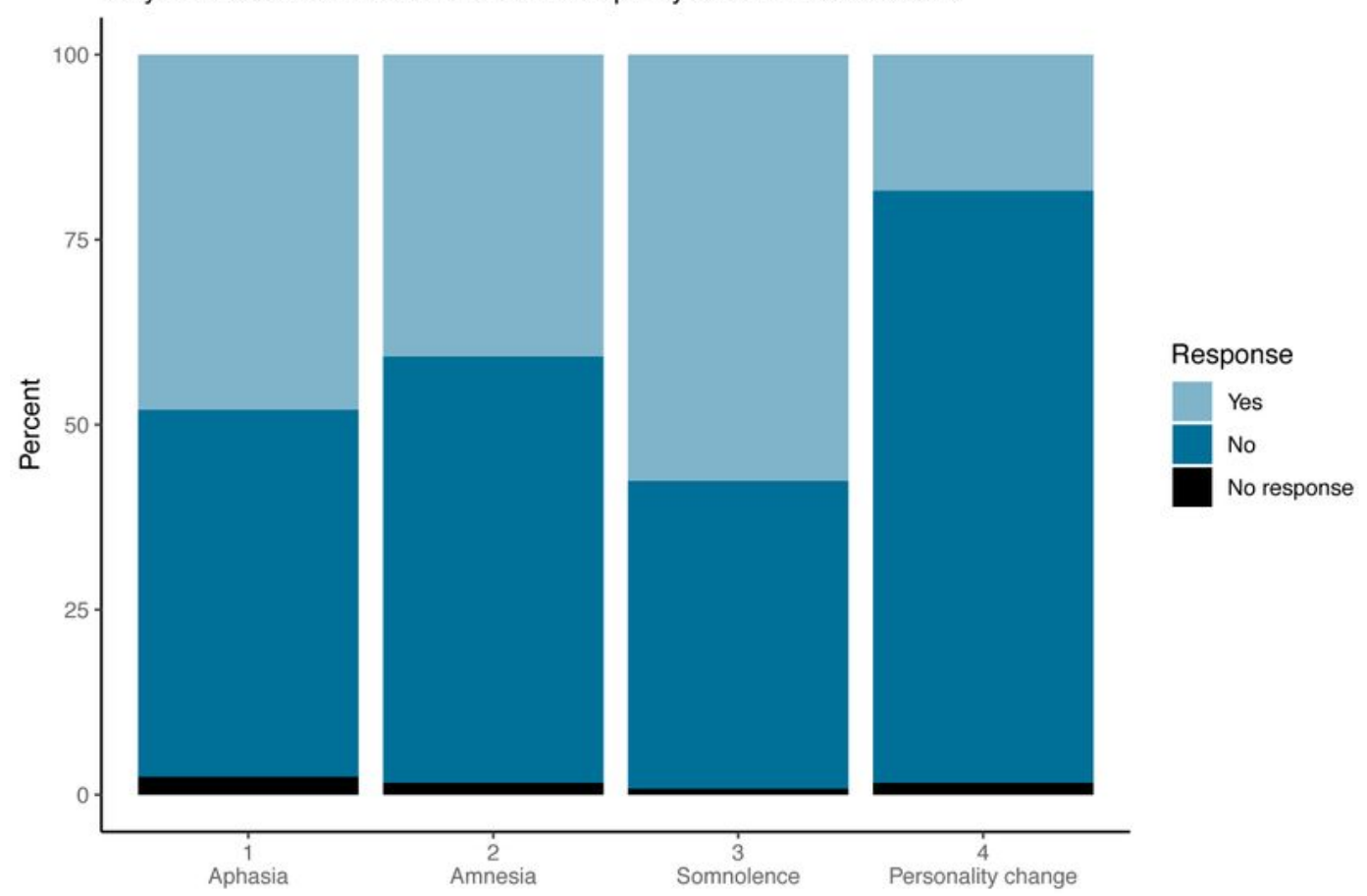

\section{B MAiD Scenarios}

If MAiD were legally available in your jurisdiction, would you support his decision to access it?

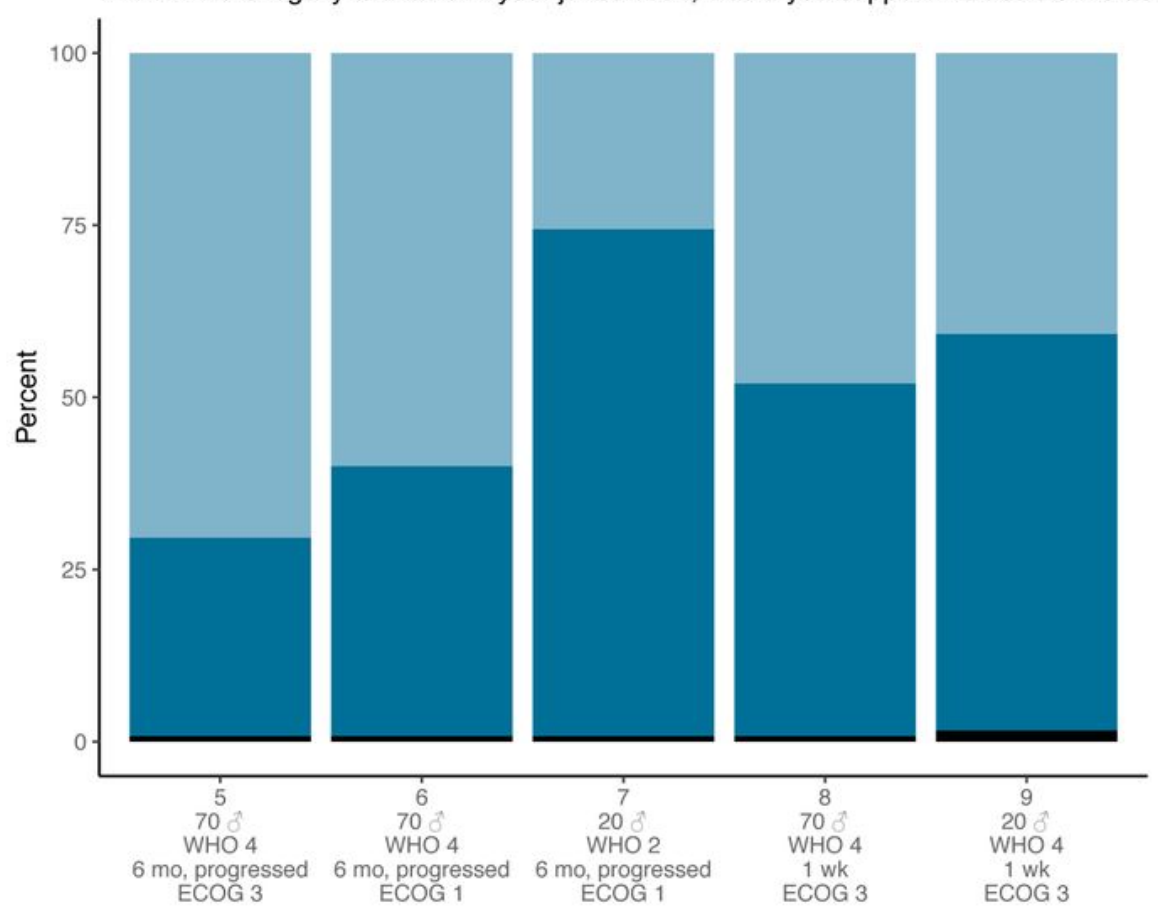

Response

Yes
No
No response

\section{Figure 3}

Responses to hypothetical scenarios. First, four scenarios (Panel A) of hypothetical glioblastoma patients with deficits in language, memory, wakefulness, or personality. Respondents were asked whether they believe the patient will have the decision-making capacity to choose whether to undergo medical assistance in dying. Second, five scenarios (Panel B) of hypothetical glioma patients with varying age, 
tumor grade, time since diagnosis, and performance status. Respondents were asked whether they would support the patient's decision to access medical assistance in dying, assuming it was legally available

\section{Supplementary Files}

This is a list of supplementary files associated with this preprint. Click to download.

- ESM1.pdf

- ESM2.pdf 